\title{
On a transfer theorem for Schur multipliers
}

\author{
By Hiroki SASAKI
}

(Received October 17, 1978)

\section{Introduction.}

In this paper we shall give an alternative proof of the following theorem proved by D. F. Holt [3].

THEOREM* (Holt).

Let $P$ be a Sylow p-subgroup of a finite group $G$, and suppose that $P$ has nilpotency class at most $p / 2$. Then the Sylow p-subgroups of the Schur multipliers of $G$ and $N_{G}(P)$ are isomorphic.

We shall prove this theorem by using the method of cohomological $G$ functors.

Maps and functors will be written on the right in their arguments, with the corresponding convention for writing composites.

Let $G$ be a finite group and $k$ a commutative ring with identity element.

DeFinition 1.

A $G$-functor over $k$ is defined to be a quadruple

$$
A=(a, \tau, \rho, \sigma),
$$

where $a, \tau, \rho, \sigma$ are families of the following kind:

$a=(a(H))$ gives, for each subgroup $H$ of $G$ (notation $H \leq G$ ), a finitely generated $k$-module $a(H)$.

$\tau=\left(\tau_{H}{ }^{K}\right)$ and $\rho=\left(\rho^{K}{ }_{H}\right)$ give, for each pair $(H, K)$ of subgroups of $G$ such that $H \leq K$, the respective $k$-homomorphisms

$$
\tau_{H}^{K}: a(H) \rightarrow a(K) \quad \text { and } \quad \rho^{K}{ }_{H}: a(K) \rightarrow a(H) .
$$

$\sigma=\left(\sigma_{H}^{g}\right)$ gives, for each pair $(H, g)$ where $H$ is a subgroup of $G$ and $g$ an element in $G$, the $k$-homomorphism

$$
\sigma_{H}^{g}: a(H) \rightarrow a\left(H^{g}\right) .
$$

These families of $k$-modules and $k$-homomorphisms must satisfy the following

Axioms for G-functors. (In these axioms, $D, H, K, L$ are any subgroups of $G ; g, g^{\prime}$ are any elements in $G$.) 
(a) $\tau_{H}^{H}=1_{a(H)}, \tau_{H}{ }^{K} \tau_{K}{ }^{L}=\tau_{H}{ }^{L}$ if $H \leq K \leq L$;

(b) $\rho^{H}{ }_{H}=1_{a(H)}, \rho^{K}{ }_{H} \rho^{H}{ }_{D}=\rho^{K}{ }_{D}$ if $K \geq H \geq D$;

(c) $\sigma_{H}^{h}=1_{a(H)}$ if $h \in H, \sigma_{H}^{g} \sigma_{H^{g}}^{g^{\prime}}=\sigma_{H}^{g g^{\prime}}$;

(d) $\tau_{H}{ }^{K} \sigma_{K}^{g}=\sigma_{H}^{g} \tau_{H^{g}} K^{g}, \rho^{K}{ }_{H} \sigma_{H}^{g}=\sigma_{K}^{g} \rho^{K^{g}}{ }_{H^{g}}$;

(e) (Mackey axiom) If $H \leq L, K \leq L$ and $\Gamma$ is a transversal of the $(H, K)$-double cosets in $L$, then

$$
\tau_{H}^{L} \rho^{L}{ }_{K}=\sum_{g \in \Gamma} \sigma_{H}^{g} \rho^{H^{g}}{ }_{H^{g} \cap K} \tau_{H^{g} \cap K}{ }^{K} .
$$

The images by the $k$-homomorphisms $\tau_{H}{ }^{K}, \rho^{K}{ }_{H}$ and $\sigma_{H}^{g}$ are simply written as follows;

$\alpha \tau_{H}{ }^{K}=\alpha^{K}$ for $\alpha$ in $a(H), \beta \rho^{K}{ }_{H}=\beta_{H}$ for $\beta$ in $a(K)$ and $\alpha \sigma_{H}^{g}=\alpha^{g}$ for $\alpha$ in $a(H)$, respectively.

A $G$-functor $A$ is naturally considered to be an $H$-functor for any subgroup $H$ of $G$. We denote such an $H$-functor by $A_{\mid H}$.

DEFINITION 2.

A $G$-functor $A=(a, \tau, \rho, \sigma)$ is called cohomological if it satisfies the following axiom $(\mathrm{C})$ :

(C) If $H \leq K \leq G$, then

$$
\rho^{K}{ }_{H} \tau_{H}{ }^{K}=|K: H| 1_{a(K)} .
$$

For examples of $G$-functors, see [2] and [8].

DEFINITION 3.

Let $A=(a, \tau, \rho, \sigma)$ be a cohomological $G$-functor and let $S$ be a subgroup of $G, \alpha$ an element in $a(S)$, and $X$ a subgroup of $G$. Then a triple $(S, \alpha, X)$ is called a singularity in $G$ for $A$ provided

(a) $\alpha_{X}^{G} \neq 0$,

(b) $\quad \alpha_{S \cap Y^{u}}=0$ for every proper subgroup $Y$ of $X$ (notation $Y<X$ ) and every element $u$ in $G$.

The subgroup $S$ is called the singular subgroup of the singularity. If the singular subgroup $S$ is a proper subgroup of $G$, then the singularity is called proper.

Now we can state a transfer theorem for cohomological $G$-functors on which our proof of Theorem* depends.

THEOREM 1.

Let $P$ be a Sylow p-subgroup of a finite group $G$ and $A=(a, \tau, \rho, \sigma)$ a cohomological $G$-functor over a commutative ring $k$. Assume that the ring $k$ is uniquely divisible by $|G: P|$ and $P$ has no proper singularity in 
$P$ for $A_{\mid P}$. Then

$$
\operatorname{Im} \rho^{G}{ }_{P}=\operatorname{Im} \rho^{N}{ }_{P}, \text { where } N=N_{G}(P) .
$$

And therefore

$$
a(G) \simeq a(N) .
$$

Let $G$ be a finite group, $M$ a right $G$-module, and let

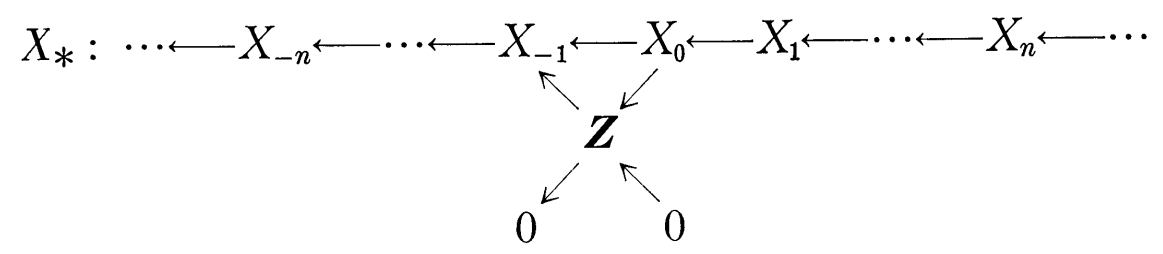

be a complete resolution of $G$, where each $G$-free module $X_{n}$ is a right $G$-module. For each subgroup $H$ of $G$, the right $G$-module $M$ and the complete resolution $X_{*}$ of $G$ are also a right $H$-module and a complete resolution of $H$, respectively.

Let $(H, K)$ be a pair of subgroups of $G$ such that $H \leq K$.

If $\Delta$ is a transversal of the $H$-left cosets in $K$, then for each element $f$ in $\operatorname{Hom}_{H}\left(X_{n}, M\right)$, we can define an element $f^{K}$ in $\operatorname{Hom}_{K}\left(X_{n}, M\right)$ by

$$
(x) f^{K}=\sum_{g \in \Delta}(x g) f g^{-1} \quad \text { for } x \text { in } X_{n} .
$$

The map $f \rightarrow f^{K}$ is a cochain morphism $\operatorname{Hom}_{H}\left(X_{n}, M\right) \rightarrow \operatorname{Hom}_{K}\left(X_{n}, M\right)$ and this morphism induces a homomorphism

$$
\operatorname{cor}_{H, K}: H^{n}(H, M) \longrightarrow H^{n}(K, M) \text {. }
$$

This homomorphism is called the corestriction from $H$ to $K$.

Every element $f$ in $\operatorname{Hom}_{K}\left(X_{n}, M\right)$ is also an element in $\operatorname{Hom}_{H}\left(X_{n}, M\right)$. If an element $f$ in $\operatorname{Hom}_{K}\left(X_{n}, M\right)$ is viewed as an element in $\operatorname{Hom}_{H}\left(X_{n}, M\right)$, we write this element $f_{H}$. The map $f \rightarrow f_{H}$ is a cochain morphism $\operatorname{Hom}_{K}\left(X_{n}, M\right) \longrightarrow \operatorname{Hom}_{H}\left(X_{n}, M\right)$ and this morphism induces a homomorphism

$$
\operatorname{res}_{K, H}: H^{n}(K, M) \longrightarrow H^{n}(H, M) \text {. }
$$

This homomorphism is called the restriction from $K$ to $H$.

For each pair $(H, g)$ of a subgroup $H$ of $G$ and an element $g$ in $G$ and for each element $f$ in $\operatorname{Hom}_{H}\left(X_{n}, M\right)$, we can define an element $f^{g}$ in $\operatorname{Hom}_{H}\left(X_{n}, M\right)$ by

$$
(x) f^{g}=\left(x g^{-1}\right) f g \quad \text { for } x \text { in } X_{n} .
$$

The map $f \rightarrow f^{g}$ is a cochain morhism $\operatorname{Hom}_{H}\left(X_{n}, M\right) \longrightarrow \operatorname{Hom}_{H^{g}}\left(X_{n}, M\right)$ and this morphism induces a homomorphism 


$$
\operatorname{con}_{H}^{g}: H^{n}(H, M) \longrightarrow H^{n}\left(H^{g}, M\right) .
$$

This homomorphism is called the conjugation by $g$.

These three homomorphisms of cohomology groups have the following properties. (In what follows $D, H, K, L$ are any subgroups of $G$ and $g, g^{\prime}$ are any elements in $G$.)

(a) $\operatorname{cor}_{H, H}=1_{H^{n}(H, M)}, \operatorname{cor}_{H, K} \operatorname{cor}_{K, L}=\operatorname{cor}_{H, L}$ if $H \leq K \leq L$;

(b) $\operatorname{res}_{H, H}=1_{H} n_{(H, M)}, \operatorname{res}_{K, H} \operatorname{res}_{H, D}=\operatorname{res}_{K, D}$ if $K \geq H \geq D$;

(c) $\operatorname{con}_{H}^{h}=1_{H^{n}(H, M)}$ if $h \in H, \operatorname{con}_{H}^{g} \operatorname{con}_{H^{g}}^{g^{\prime}}=\operatorname{con}_{H}^{g g^{\prime}}$;

(d) $\operatorname{cor}_{H, K} \operatorname{con}_{K}^{g}=\operatorname{con}_{H}^{g} \operatorname{cor}_{H^{g}, K^{g}}, \operatorname{res}_{K, H} \operatorname{con}_{H}^{g}=\operatorname{con}_{K}^{g} \operatorname{res}_{K^{g}, H^{g}}$;

(e) If $H \leq L, K \leq L$ and $\Gamma$ is a transversal of the $(H, K)$-double cosets in $L$, then

$$
\operatorname{cor}_{H, L} \operatorname{res}_{L, K}=\sum_{g \in I} \operatorname{con}_{H}^{g} \operatorname{res}_{H^{g}, H^{g} \cap K} \operatorname{cor}_{H^{g} \cap K, K} ;
$$

(f) If $H \leq K$, then

$$
\operatorname{res}_{K, H} \operatorname{cor}_{H, K}=|K: H| 1_{H^{n}(K, M)} .
$$

Note that the axioms for the cohomological $G$-functors are abstracted from these properties.

Let $M(G)$ denote the Schur multiplier $H^{2}\left(G, C^{*}\right)$ of a finite group $G$. For each subgroup $H$ of $G$, put $a(H)=\Omega_{1}\left(M(H)_{p}\right)$, where $M(H)_{p}$ is the Sylow $p$-subgroup of $M(H)$ and $\Omega_{1}\left(M(H)_{p}\right)$ is the subgroup of $M(H)_{p}$ generated by the elements of order $p$. Then $a(H)$ is a finite dimensional $\boldsymbol{F}_{p}$-module. For each pair $(H, K)$ of subgroups of $G$ such that $H \leq K$, let $\tau_{H}^{K}$ and $\rho^{K}{ }_{H}$ be $\operatorname{cor}_{H, K \mid a(H)}$ and $\operatorname{res}_{K, H \mid a(K)}$, respectively. For each pair $(H, g)$ of a subgroup $H$ of $G$ and an element $g$ in $G$, we define $\sigma_{H}^{g}=\operatorname{con}_{H \mid a(H)}^{g}$. Then $A=(a, \tau, \rho, \sigma)$ is a cohomological $G$-functor over $\boldsymbol{F}_{p}$. We call this functor the multiplier functor (with respect to a prime $p$ ).

If a Sylow $p$-subgroup $P$ of $G$ has no proper singularity in $P$ for the multiplier functor, then by Theorem 1 we have

$$
\Omega_{1}\left(M(G)_{p}\right) \simeq \Omega_{1}\left(M\left(N_{G}(P)\right)_{p}\right) .
$$

Hence by Tate's theorem it follows that

$$
M(G)_{p} \simeq M\left(N_{G}(P)\right)_{p} .
$$

We shall establish Theorem* (Holt) by proving the following Theorem 2.

THEOREM 2.

Let $P$ be a p-group of nilpotency class at most $p / 2$. Then $P$ has no 
proper singularity in $P$ for the multiplier functor.

\section{Acknowledgment}

The author thanks to Dr. Yoshida for this theme and for his helpful advices.

\section{A transfer theorem for cohomological $G$-functors.}

In this section we shall prove Theorem 1 .

Let $G$ be a finite group and let $A=(a, \tau, \rho, \sigma)$ be a cohomological $G$ functor over a commutative ring $k$.

Lemma 1.

Let $H$ be a subgroup of $G$ such that the ring $k$ is uniquely divisible by $|G: H|$. Then the k-homomorphism $\rho_{H}^{G}: a(G) \rightarrow a(H)$ is a monomorphism and the k-homomorphism $\tau_{H}^{G}: a(H) \rightarrow a(G)$ is an epimorphism.

Moreover

$$
a(H)=\operatorname{Im} \rho^{G} \oplus \operatorname{Ker} \tau_{H}^{G} .
$$

Proof. The composition homomorphism $\rho_{H}^{G} \tau_{H}{ }^{G}: a(G) \rightarrow a(G)$ is equal to $|G: H| 1_{a(G)}$ and this is an automorphism of $a(G)$ since the ring $k$ is uniquely divisible by $|G: H|$. The lemma easily follows from this fact.

LEMMA 2.

Let $(S, \alpha, X)$ be a singularity in $G$ for $A$. Then the following hold.

(1) For every elements $g, h$ in $G$, a triple $\left(S^{g}, \alpha^{g}, X^{h}\right)$ is also a singularity in $G$ for $A$.

(2) There exists an element $g$ in $G$ such that $X^{g} \leq S$.

(3) If the ring $k$ is uniquely divisible by $|S: H|$ for a subgroup $H$ of $S$, then $\left(H, \alpha_{H}, X\right)$ is also a singularity in $G$ for $A$.

(4) If a subgroup $R$ of $G$ contains $S$, then $\left(R, \alpha^{R}, X\right)$ is also a singularity in $G$ for $A$.

(5) If a subgroup $L$ of $G$ contains $S$, then there exists an element $g$ in $G$ such that $\left(S, \alpha, X^{g}\right)$ is a singularity in $L$ for $\left.A\right|_{L}$.

(6) If a subgroup $L$ of $G$ contains $X$, then $\left(S^{g} \cap L, \alpha^{g}{ }_{S^{g} \cap L}, X\right)$ is a singularity in $L$ for $\left.A\right|_{L}$ for some element $g$ in $G$. If moreover $G=L S$, then $\left(S \cap L, \alpha_{S \cap L}, X\right)$ is a singularity in $L$ for $A_{1 L}$.

Proof. (1). This follows immediately from Definition 3 .

(2). Let $\Gamma$ be a transversal of the $(S, X)$-double cosets in $G$, then by Mackey axiom 


$$
\alpha_{X}^{G}=\sum_{g \in \Gamma}\left(\alpha_{S^{g} \cap X}\right)^{X}
$$

Thus there exists an element $g$ in $G$ such that $\alpha_{S \cap X^{g}} \neq 0$ since $\alpha_{X}^{G} \neq 0$. If $S \cap X^{g}<X^{g}$, then by Definition 3 we have $\alpha_{S \cap X^{g}}=0$, a contradiction. So $X^{g} \leq S$.

(3). Since $A$ is a cohomological $G$-functor, we have

$$
\begin{aligned}
\left(\alpha_{H}^{G}\right)_{X} & =\left(\alpha_{H}^{S}\right)^{G}{ }_{X} \\
& =|S: H| \alpha^{G}{ }_{X} \\
& \neq 0 .
\end{aligned}
$$

For every proper subgroup $Y$ of $X$ and every element $u$ in $G$, we have

$$
\begin{aligned}
\left(\alpha_{H}\right)_{H \cap Y^{u}} & =\left(\alpha_{S \cap Y^{u}}\right)_{H \cap Y^{u}} \\
& =0 .
\end{aligned}
$$

Thus $\left(H, \alpha_{H}, X\right)$ is a singularity in $G$ for $A$.

(4). It is clear that $\left(\alpha^{R}\right)^{G} \neq 0$.

Let $Y$ be a proper subgroup of $X$ and $u$ an element in $G$. If $\Gamma$ is a transversal of the $\left(S, R \cap Y^{u}\right)$-double cosets in $R$, then by Mackey axiom

$$
\begin{aligned}
\alpha_{R \cap Y^{u}}^{R} & =\sum_{g \in \Gamma}\left(\alpha_{\left.S^{g} \cap R \cap Y^{u}\right)^{R \cap Y^{u}}}\right. \\
& =\sum_{g \in \Gamma}\left(\left(\alpha_{\left.S \cap R^{g^{-1}} \cap Y^{u g-1}\right)^{g}}\right)^{R \cap Y^{u}}\right. \\
& =0 .
\end{aligned}
$$

Thus $\left(R, \alpha^{R}, X\right)$ is a singularity in $G$ for $A$.

(5). Since $\left(\alpha^{L}\right)^{G}{ }_{X}=\alpha_{X}^{G} \neq 0$, there exists an element $g$ in $G$ such that $\alpha^{L}{ }_{L \cap X^{g}} \neq 0$ by Mackey axiom. Then again by Mackey axiom there exists an element $s$ in $S$ such that $\alpha_{S \cap L^{s} \cap X^{g s}} \neq 0$. Thus by Definition 3 we have $X^{g} \leq L$ and hence $\alpha^{L} X^{g} \neq 0$. It is clear that $\alpha_{S \cap Z^{u}}=0$ for every proper subgroup $Z$ of $X^{g}$ and every element $u$ in $L$. Thus $\left(S, \alpha, X^{g}\right)$ is a singularity in $L$ for $\left.A\right|_{L}$. (6). Since $\left(\alpha^{G}\right)_{X}=\alpha^{G}{ }_{X} \neq 0$, there exists an element $g$ in $G$ such that $\left(\alpha^{g}{ }_{S^{g} \cap L}\right)^{L}{ }_{X}$ $\neq 0$ by Mackey axiom. For every proper subgroup $Y$ of $X$ and every element $u$ in $L$, we have

$$
\begin{aligned}
\left(\alpha^{g} S^{g} \cap L\right)_{S^{g} \cap L \cap Y}^{u} & =\alpha^{g} S^{g} \cap L \cap Y^{u} \\
& =\left(\alpha_{\left.S \cap\left(L \cap Y^{u}\right)^{g-1}\right)^{g}}\right. \\
& =0 .
\end{aligned}
$$

Thus $\left(S^{g} \cap L, \alpha^{g}{ }^{g} \cap L, X\right)$ is a singularity in $L$ for $A_{I L}$. When $G=L S$, we can take $g=1$ so that $\left(S \cap L, \alpha_{S \cap L}, X\right)$ is a singularity in $L$ for $A_{\mid L}$. The lemma is proved. 
The following lemma gives us a technique for proving Theorem 1 .

LEMMA 3.

Let $H$ be a subgroup of $G$ such that the ring $k$ is uniquely divisible by $|G: H|, R$ a subgroup of $H$, and let $B$ be a k-submodule of $a(R)$. Assume that

$$
\operatorname{Im} \rho_{R}^{G}<B \leq \operatorname{Im} \rho^{H} .
$$

Then the following hold.

(1) There exists an element $\alpha$ in $a(H)$ such that $\alpha \neq 0, \alpha^{G}=0$, and $0 \neq \alpha_{R} \in B$.

(2) Let $X$ be a subgroup of $R$ such that $\alpha_{X} \neq 0$ and $\alpha_{H \cap Y^{u}}=0$ for every proper subgroup $Y$ of $X$ and every element $u$ in $G$. Then there exists an element $g$ in $G-H$ such that

(a) $\left(H \cap H^{g}, \alpha_{H \cap H^{g}}^{g}-\alpha_{H \cap H^{g}}, X\right)$ is a singularity in $H$ for $A_{\mid H}$; and

(b) $\left(R \cap H^{g}, \alpha^{g} R_{\cap H^{g}}-\alpha_{R \cap H^{g}}, X\right)$ is a singularity in $R$ for $A_{1 R}$.

Proof. (1). By Lemma 1 it follows that

$$
a(H)=\operatorname{Im} \rho_{H}^{G} \oplus \operatorname{Ker} \tau_{H}^{G} .
$$

Thus we have

$$
\operatorname{Im} \rho^{H}{ }_{R}=\operatorname{Im} \rho^{G}{ }_{R} \oplus\left(\operatorname{Ker} \tau_{H}^{G}\right) \rho^{H} .
$$

Hence by our assumption on $B$ it follows that

$$
B \cap\left(\operatorname{Ker} \tau_{H}^{G}\right) \rho_{R}^{H} \neq 0 .
$$

Namely there exists an element $\alpha$ in $a(H)$ such that $\alpha \neq 0, \alpha^{G}=0$, and $0 \neq \alpha_{R} \in B$ as required.

(2). Let $\Gamma$ be a transversal of the $(H, H)$-double cosets in $G$. Then

$$
\begin{aligned}
\sum_{g \in \Gamma}\left(\alpha^{g} H \cap H^{g}\right. & \left.-\alpha_{H \cap H^{g}}\right)_{X}^{H} \\
& =\left(\sum_{g \in \Gamma} \alpha^{g}{ }_{H \cap H^{g}}\right)_{X}-\left(\sum_{g \in \Gamma} \alpha_{H \cap H^{g} H}\right)_{X} \\
& =\alpha^{G}{ }_{X}-|G: H| \alpha_{X} \\
& \neq 0 .
\end{aligned}
$$

Thus there exists an element $g$ in $G-H$ such that

$$
\left(\alpha_{H \cap H^{g}}^{g}-\alpha_{H \cap H^{g}}\right)^{H}{ }_{X} \neq 0 .
$$

By our assumption on the subgroup $X$ we have

$$
\left(\alpha_{H \cap H^{g}}-\alpha_{H \cap H^{g}}\right)_{H \cap H^{g} \cap Y^{u}}=0
$$


for every proper subgroup $Y$ of $X$ and every element $u$ in $H$. Thus $\left(H \cap H^{g}, \alpha_{H \cap H^{g}}-\alpha_{H \cap H^{g}}, X\right)$ is a singularity in $H$ for $A_{\mid H}$.

By Lemma $2(6)$ there exists an element $h$ in $H$ such that $\left(R \cap\left(H \cap H^{g}\right)^{h},\left(\alpha^{g} H_{\cap} H^{g}-\alpha_{\left.\left.\left.H \cap H^{g}\right)^{h}{ }_{R \cap\left(H \cap H^{g}\right.}\right)^{n}, X\right)}\right.\right.$ is a singularity in $R$ for $A_{\mid R}$. Since $R \cap\left(H \cap H^{g}\right)^{h}=R \cap H^{g h}$ and $\left(\alpha^{g}{ }_{H \cap H^{g}}-\alpha_{H \cap H^{g}}\right)^{h}{ }_{R \cap\left(H \cap H^{g}\right)^{h}}=\alpha^{g h}{ }_{R \cap H^{g h}}-\alpha_{R \cap H^{g h}}$, we have that $\left(R \cap H^{g}, \alpha^{g}{ }_{R \cap H^{g}}-\alpha_{R \cap H^{g}}, X\right)$ is a singularity in $R$ for $A_{\mid R}$ by replacing $g$ with $g^{-1} h$ if necessary. The lemma is proved.

Remark. Let $G, H, R$, and $\alpha$ be as in Lemma 3. Assume that for every subgroup $Q$ of $R$ and every element $g$ in $G$, there exist a subgroup $T$ of $R$ and an element $h$ in $H$ such that $H \cap Q^{g} \leq T^{h}$. Then a subgroup $X$ of minimal order of $R$ such that $\alpha_{X} \neq 0$ satisfies the assumption of Lemma 3 (2). Because for a proper subgroup $Y$ of $X$ and an element $g$ in $G$, there exist a subgroup $T$ of $R$ and an element $h$ in $H$ such that $H \cap Y^{g} \leq T^{h}$. Hence $H \cap Y^{g h^{-1}} \leq T \leq R$. Since $h \in H$ and $\alpha \in a(H)$, we have $\alpha_{H \cap Y^{g}}=\alpha_{H \cap Y^{g h-1}}$. Thus by the minimality of the order of $X$ it follows that $\alpha_{H \cap Y^{g}}=0$.

Theorem 1.

Let $P$ be a Sylow p-subgroup of a finite group $G$ and $A=(a, \tau, \rho, \sigma)$ a cohomological $G$-functor over a commutative ring $k$. Assume that the ring $k$ is uniquely divisible by $|G: P|$ and $P$ has no proper singularity in $P$ for $A_{1 P}$. Then

$$
\operatorname{Im} \rho_{P}^{G}=\operatorname{Im} \rho^{N}{ }_{P}, \text { where } N=N_{G}(P) .
$$

And therefore

$$
a(G) \simeq a(N) .
$$

Proof. Suppose that $\operatorname{Im} \rho^{G}{ }_{P}<\operatorname{Im} \rho^{N}{ }_{P}$. Then by Lemma 3 there exists an element $\alpha$ in $a(N)$ such that $\alpha \neq 0, \alpha^{G}=0$, and $\alpha_{P} \neq 0$. Take a subgroup $X$ of minimal order of $P$ such that $\alpha_{X} \neq 0$. Then again by Lemma 3 there exists an element $g$ in $G-N$ such that $\left(P \cap N^{g}, \alpha_{P \cap N^{g}}^{g}-\alpha_{P \cap N^{g}}, X\right)$ is a singularity in $P$ for $A_{1 P}$. Then we have $P \cap N^{g}=P$ by our assumption on $P$. Hence it must hold that $P=P^{g}$, a contradiction. Thus we have

$$
\operatorname{Im} \rho_{P}^{G}=\operatorname{Im} \rho^{N}{ }_{P}
$$

The homomorphism $\rho^{G}{ }_{N}$ gives an isomorphism of $a(G)$ to $a(N)$ since $\rho^{G}{ }_{N}$ and $\rho^{N}{ }_{P}$ are monomorphisms and $\rho^{G}{ }_{P}=\rho^{G}{ }_{N} \rho^{N}{ }_{P}$. Theorem 1 is proved. 


\section{The proof of Theorem 2 .}

In this section we shall prove Theorem 2 and Theorem*.

THEOREM 2.

Let $P$ be a p-group of nilpotency class at most $p / 2$. Then $P$ has no proper singularity in $P$ for the multiplier functor.

Proof. Suppose $P$ has a proper singulaity $(S, \alpha, X)$ in $P$ for the multiplier functior. By Lemma 2 we may assume that the singular subgroup $S$ is a maximal subgroup of $P$ and $X$ is contained in $S$.

Let

$$
1 \longrightarrow R \longrightarrow F \longrightarrow P \longrightarrow 1
$$

be a free presentation of $P$ and let $F_{S}$ be the complete inverse image of $S$ in $F$. The commutator subgroup $\left[F_{\mathrm{S}}, R\right]$ of $F_{S}$ and $R$ is normal in $F$ since $S$ is normal in $P$. Thus we have two extensions

$$
1 \longrightarrow \bar{R} \longrightarrow \bar{F} \longrightarrow P \longrightarrow 1 \text { and } 1 \longrightarrow \bar{R} \longrightarrow \bar{F}_{S} \longrightarrow S \longrightarrow 1
$$

where bars denote images modulo $\left[F_{S}, R\right]$. The latter extension is a central extension of $S$ of free type. It is well known that $\bar{D}=\bar{R} \cap \bar{F}_{S}^{\prime}$ is the torsion subgroup of $\bar{R}$ and $\bar{D} \simeq M(S)$. There exists a subgroup $\bar{J}$ of $\bar{R}$ such that $\bar{R}=\bar{J} \times \bar{D}$ as $\bar{R}$ is finitely generated. Thus we have a central extension of $S$

$$
1 \longrightarrow Z \longrightarrow K \longrightarrow S \longrightarrow 1
$$

where $Z=\bar{R} / \bar{J} \simeq \bar{D}$ and $K=\bar{F}_{S} / \bar{J}$. In the Hochschild - Serre exact sequence

$$
1 \longrightarrow \operatorname{Hom}\left(S, C^{*}\right) \longrightarrow \operatorname{Hom}\left(K, C^{*}\right) \longrightarrow \operatorname{Hom}\left(Z, C^{*}\right) \longrightarrow M(S)
$$

associated to this central extension of $S$, the transgression map

$$
t: \operatorname{Hom}\left(Z, C^{*}\right) \longrightarrow M(S)
$$

is an isomorphism. For the proof of this fact, see [7] $\S 1, \S 3$ or [4] Kap. $\mathrm{V} \S 23$ or [5] Ch. $2 \S 7, \S 9$. Hence there exists a unique element $\phi$ in $\Omega_{1}\left(\operatorname{Hom}\left(Z, C^{*}\right)\right)$ such that

$$
\alpha=(\phi) t .
$$

The factor group $P / S$ acts on $Z$ and therefore on $\operatorname{Hom}\left(Z, C^{*}\right)$. On the other hand $P / S$ acts on $M(S)$. These operations by $P / S$ are commutative with the transgression $t$. Let $u$ be an element in $P-S$. Then by Mackey axiom

$$
\alpha_{X}^{P}=\sum_{i=0}^{p-1} \alpha^{u^{i}}{ }_{X}
$$


Therefore

$$
\left(\sum_{i=0}^{p-1} \phi^{u^{i}}\right) t=\sum_{i=0}^{p-1} \alpha^{u^{i}} \neq 0 .
$$

Let $\phi=\sum_{i=0}^{p-1} \phi^{u^{i}}$, then the order of $\phi$ is $p$. Let $I$ be the kernel of $\phi$, then it follows that $\bigcap_{i=1}^{p-1} I^{u^{i}} \leq \operatorname{Ker} \psi$. On the other hand we have $[Z, u] \leq \operatorname{Ker} \psi$. Suppose $I=I^{u}$, then $I=\operatorname{Ker} \psi$ so that $[Z, u] \leq I$. Thus $\phi=\phi^{u}$ and hence $\phi=p \phi=0$, a contradiction. Therefore $I \neq I^{u}$. Hence if we put $L=\bigcap_{i=0}^{p-1} I^{u^{i}}$, then the factor group $Z / L$ is an elementary abelian $p$-group of order $p^{p}$ that has a basis on which $u$ acts regularly. Let $T$ be the semidirect product of $Z$ by $P$. Since $L$ is normalized by $P$, the semidirect product $T$ involves the wreath product $\boldsymbol{Z}_{p}$ wr $\boldsymbol{Z}_{p}$ so that the nilpotency class of $T$ is at least $p$.

On the other hand as in [3] Lemma 7 it follows that the nilpotency class of $T$ is less than $p$ by using the assumption that $P$ has nilpotency class at most $p / 2$. Thus we have a contradiction. Theorem 2 is proved.

Proof of Theorem* (Holt). Theorem 1 and Theorem 2 imply that if a Sylow $p$-subgroup $P$ of $G$ is of nilpotency class at most $p / 2$, then

$$
\left(\Omega_{1}\left(M(G)_{p}\right)\right) \operatorname{res}_{G, P}=\left(\Omega_{1}\left(M(N)_{p}\right)\right) \operatorname{res}_{N, P}, \text { where } N=N_{G}(P) .
$$

Since $M(P)=\operatorname{Im} \operatorname{res}_{G, P} \oplus \operatorname{Ker} \operatorname{cor}_{P, G}$, we have

$$
\left(M(N)_{p}\right) \operatorname{res}_{N, P}=\left(M(G)_{p}\right) \operatorname{res}_{G, P} \oplus\left(\left(M(N)_{p}\right) \operatorname{res}_{N, P} \cap \operatorname{Ker} \operatorname{cor}_{P, G}\right) .
$$

Hence by the first equation it follows that

$$
\left(M(N)_{p}\right) \operatorname{res}_{N, P} \cap \operatorname{Ker} \operatorname{cor}_{P, G}=0
$$

so that

$$
\left(M(G)_{p}\right) \operatorname{res}_{G, P}=\left(M(N)_{p}\right) \operatorname{res}_{N, P} .
$$

As in the proof of Theorem $1 \operatorname{res}_{G, N \mid M(G)}$ gives an isomorphism of $M(G)_{p}$ to $M(N)_{p}$.

REMARK. As we have seen in the proof of Theorem 2, if a p-group $P$ has a proper singularity in $P$ for the multiplier functor, then $P$ has a maximal subgroup $S$ whose Schur multiplier $M(S)$ has a factor group isomorphic to an elementary abelian $p$-group of order $p^{p}$. Therefore a $p$-group which has no such maximal subgroup has no proper singularity. For example a 2 -group of miximal class has no proper singularity. However it is 
still open to determine a necessary and sufficient condition for a $p$-group to have no proper singularity for the multiplier functor.

\section{References}

[1] A. Babakhanian: Cohomological Methods in Group Theory, Marcel Dekker, Inc., New York, 1972.

[2] J. A. GreEn: Axiomatic representation theory for finite groups, J. Pure Appl. Algebra, 1 (1971), 41-77.

[3] D. F. Holt : On the local control of Schur multipliers, Quart. J. Math. Oxford (2), 28 (1977), 495-508.

[4] B. HupPerT : Endliche Gruppen I, Springer-Verlag, Berlin/Hidelberg/New York, 1967.

[5] M. Suzuki: Group Theory, Iwanami, Tokyo, 1977 (Japanese).

[6] E. WeIss : Cohomology of Groups, Academic Press, New York and London, 1969.

[7] K. YAMAZAKI: Projective representations and ring extensions, J. Fac. Sci. Univ. Tokyo. Sect. 1 vol. 10 (1964), 147-195.

[8] T. Yoshida: On $G$-functors (I): Transfer theorems for cohomological $G$-functors, Hokkaido Math. J., to appear.

[9] T. Yoshida: Transfer theorems in cohomology theory of finite groups, Japanese 23-th Symposium on Algebra, 1977.

[10] T. YoshidA: Character-theoretic transfer, J. Algebra, 52 (1978), 1-38.

Hiroki SASAKI

Department of Mathematics

Faculty of Science

Hokkaido University

Sapporo, 060 Japan 